\title{
Repair of ventricular septal defect through anterolateral thoracotomy with central cannulation: our experience
}

\author{
Sunil Dixit ${ }^{1} \cdot$ Anil Sharma ${ }^{1} \cdot$ Jaikishan Suthar ${ }^{1} \cdot$ Vikram Watti $^{1} \cdot$ Mohit Sharma $^{1}$ (i) \\ Received: 21 May 2019 / Revised: 14 January 2020 / Accepted: 27 January 2020 / Published online: 16 March 2020 \\ (C) Indian Association of Cardiovascular-Thoracic Surgeons 2020
}

\begin{abstract}
Background At present thoracotomy with femoro-femoral bypass is an established approach for minimally invasive open heart surgeries, but thoracotomy with conventional cannulation is yet to be established. We performed 54 cases of ventricular septal defect (VSD) closure via anterolateral thoracotomy approach with central cannulation. Here we are describing our results and experience of VSD closure via anterolateral thoracotomy approach.

Aim and objective The aim of our study was to evaluate early outcomes of VSD repair via anterolateral thoracotomy with central cannulation.

Methods This is a retrospective, observational, descriptive type of study. Fifty four patients (31 males, 23 females) underwent VSD repair from November 2016 to November 2018 via anterolateral thoracotomy with age ranges from 3 to 22 years (mean age $10.57 \pm 8.88$ years). Mean body weight was $22.29 \pm 13.44 \mathrm{~kg}$ (range 10 to $48 \mathrm{~kg}$ ). The VSD was perimembranous in 47 patients, subpulmonic in 2, muscular in 2 , and inlet in 3 patients.

Results There was no operative or late mortality. The mean incision length was $7.16 \pm 02.08 \mathrm{~cm}$ (range, $5 \mathrm{~cm}$ to $9 \mathrm{~cm}$ ). Average duration of cardiopulmonary bypass (CPB) was $61.72 \pm 14.20 \mathrm{~min}$ (range, 48-78 min), and aortic cross-clamp time was 38.51 \pm $13.08 \mathrm{~min}$ (range, $26-56 \mathrm{~min}$ ). The average postoperative intensive care unit (ICU) stay was $1.83 \pm 1.32$ days (range, $1-3$ days), and hospital stay was $4.92 \pm 1.82$ days (range, $4-7$ days).

Conclusion Anterolateral thoracotomy with conventional central cannulation can be a safe alternative to median sternotomy with superior cosmetic results for the repair of VSDs.
\end{abstract}

Keywords Ventricular septal defect · Central cannulation · Anterolateral thoracotomy

\section{Introduction}

Median sternotomy has been considered as the standard approach for congenital heart defect (CHD) repair, with

Mohit Sharma

aries.mohit@gmail.com

Sunil Dixit

drsunil_dixit@yahoo.com

Anil Sharma

anilsharma.1961@yahoo.com

Jaikishan Suthar

dr.jksuthar@gmail.com

Vikram Watti

how_r_u9@ rediffmail.com

1 Department of Cardio-Vascular and thoracic surgery, S.M.S. Medical College and Group of Hospitals, Jaipur, India excellent results since the beginning of cardiac surgery [1]. Several minimally invasive approaches, including ministernotomy $[2,3]$, a transxiphoid approach without sternotomy, anterolateral thoracotomy $[4,5]$, and posterolateral thoracotomy [6], have been developed to achieve favorable cosmetic results with a perfect repair. As the clinical results of CHD operations improve (with near zero perioperative mortality), the cosmetic implications become more important in the evaluation of these procedures. Potential advantages include improved cosmetic results and comfort for the patient, as well as shorter hospital stays that reduce total costs. Nevertheless, whether minimally invasive approaches actually reduce postoperative pain and bleeding and improve respiratory function is still a matter of controversy. This retrospective study reviews our experience with the use of right anterolateral thoracotomy and conventional central cannulation for VSD repair in 54 patients. Anterolateral thoracotomy with central cannulation has already been used at few centers 
worldwide for radical correction of congenital heart diseases [7].

\section{Methodology}

This is a retrospective, observational, descriptive type of study. Intraoperative events and operative outcomes with time were evaluated by contingency table analysis. Every patient was asked to fill a pre-formed subjective questionnaire about surgical scar and return to routine work in 1-month follow-up, and photographs were taken after consent of patients.

\section{Demographic characteristics}

This study included 54 patients (23females, 31 males) who underwent VSD closure via anterolateral thoracotomy approach from November 2016 to November 2018 (Table 1). Mean age was $10.57 \pm 8.88$ years (age range 3 to 22 years), and body weight was $22.29 \pm 13.44 \mathrm{~kg}$ (weight range 10 to $48 \mathrm{~kg}$ ). Subpulmonary VSD was present in 2 patients, inlet VSD in 3, and muscular VSD in 2, and perimembranous VSD was present in 47 patients. Other concomitant defects included atrial septal defect in 4 patients and tricuspid insufficiency in 2 patients, and prolapse of the right aortic cusp was present in 2 patients.

\section{Inclusion criteria}

a. All types of VSDs except exclusion criteria

\section{Exclusion criteria}

a. Patients with right ventricular outflow tract obstruction

b. Atrioventricular canal defect

c. Ischemic ventricular septal rupture detected by preoperative echocardiography

d. Swiss cheese VSD (Multiple VSDs)

e. Apical VSD

f. Doubly committed VSD

g. Re-do cases

h. VSD associated with aortic regurgitation and patent ductus

i. VSD associated with complex congenital cardiac defects in 2-D echo (i.e., transposition of great arteries/TGA)

\section{Operative procedure}

\section{Anesthesia}

We used the standard protocol of anesthesia, i.e., as for a conventional VSD closure under general anesthesia.
The patients were intubated with a single lumen endotracheal tube.

\section{Positioning the patient}

The patient was placed in a supine position, and a sand sack was placed under the right scapula to allow the patient's right chest to be elevated slightly $\left(30-40^{\circ}\right)$ according to the need of the surgeon for a better working field exposure (Fig. 1a). The patient's right arm was positioned besides the body. Right groin was prepared and draped for emergency situation.

\section{Thoracotomy}

A 5-9-cm sub-mammary incision was made in the right submammary fold starting 3 to $4 \mathrm{~cm}$ apart from the lateral border of the sternum (Figs. 1 and 2). The breast tissue was gently mobilized, and the right thoracic cavity was entered through the third or fourth intercostal space. We preferred third space, if aorta was a priority to approach for cannulation like in large right atrium (RA) cases, and we preferred fourth space in cases where we wanted to approach inferior vena cava (IVC) first for easy looping prior to going on bypass. The decision of space was taken by chest X-ray prior to the exposure of surgical field. In chest X-ray, if RA was enlarged and aorta was difficult to approach, then we preferred third space for easy aortic cannulation. After putting a single venous cannula, we first went on bypass to decompress the heart for more space and then introduced second venous cannula to achieve full

Table 1 Demographic characteristics and patient diagnoses

\begin{tabular}{ll}
\hline Variable & Mean \pm SD (range) or no \\
\hline Age & $10.57 \pm 8.88$ \\
Sex & \\
1. Male & 31 \\
2. Female & 23 \\
Body weight & $22.29 \pm 13.44$ \\
Type of VSD & \\
1. Perimembranous VSD & 47 \\
2. Subpulmonic VSD & 2 \\
3. Muscular VSD & 2 \\
4. Inlet VSD & 3 \\
Associated defects & \\
1. Atrial septal defect/patent foramen ovale & 4 \\
2. Tricuspid insufficiency & 2 \\
3. Aortic cusp prolapsed* & 2 \\
\hline
\end{tabular}

Continuous variables expressed as mean \pm standard deviation

*In our pre-op echo, significant aortic cups prolapse was not mentioned but intra-op, and we found mild aortic cusp prolapse, and after repair of VSD, there was no significant aortic regurgitation, so we did not repair or replace aortic valve 
Fig. 1 a Thoracotomy position. b Operative exposure
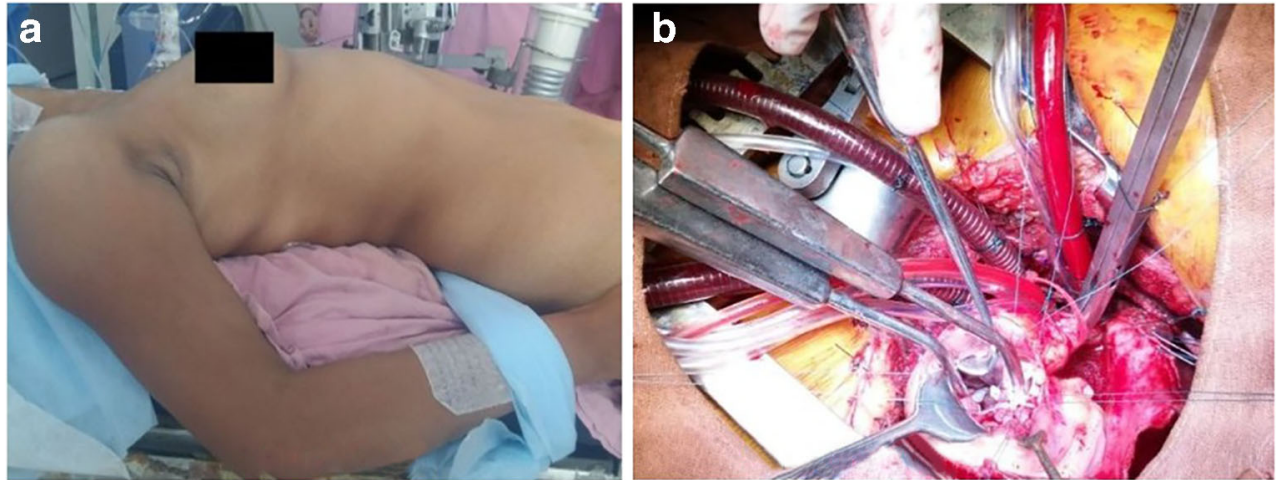

flows, followed by superior vena cava (SVC) and IVC looping afterward. In most of the cases, we approached fourth space to gain easy approach to IVC. A chest retractor was placed in between the intercostal space and opened gradually and gently so that ribs were protected. The right lung was compressed with a wet sponge to expose the pericardial sac. The pericardial sac was entered through an incision 3-4-cm anterior and parallel to the phrenic nerve extending from the diaphragm to the aortic reflection. Multiple pericardial stay sutures were placed in a manner to lift the heart into the operative field.

\section{Cannulation, bypass, and technical challenges}

The most important part of this procedure was good surgical exposure. All the operations were performed with the standard cardiopulmonary bypass through central cannulation with moderate hypothermia. Aortic and venous cannulation was same as in standard sternotomy. We used long snares
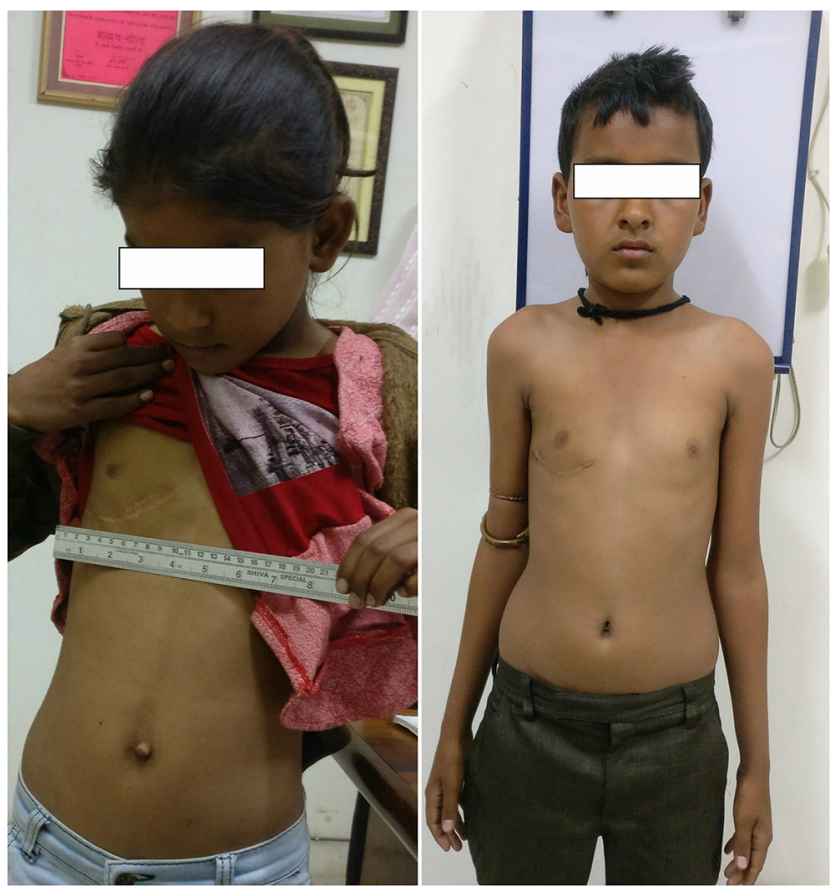

Fig. 2 Postoperative surgical scar
(Fig. 1b) so that the snares and artery forceps attached with snares would not come in the operating field and exposure was good. Double purse string sutures were applied for both aortic and cardioplegia cannulae. For good surgical exposure

1. Thoracotomy space (third or fourth from right anterolateral thoracotomy and sometimes second intercostal space for left anterolateral thoracotomy) was decided preoperatively by chest X-ray.

2. After thoracotomy, pericardium was hitched by silk stay sutures sequentially and gradually (step by step). Traction on stay sutures was given outside and downward so that the cardiac surface came in surgeon's view (Fig. 1b).

3. Aortic cannulation was the difficult part. We used Medtronic arterial (Flexible Arch Arterial Cannula, Manufacturer, Medtronic, Inc. Minneapolis, USA) and Edward life sciences venous cannula (Thin-Flex Single Stage Venous Drainage Cannula, Manufacturer, Edward Life sciences LLC, USA).

4. For aortic cannulation, we grasped the epiaortic tissues by right angled artery forceps for gentle traction, and we held the aortic cannula tip by another artery forceps for smooth insertion.

5. If there was any difficulty in venous cannulation (in large RA cases), then we first went on bypass with single cannula to get enough space for a free access to SVC and IVC.

6. For aortic cross clamping, we used Chitwood clamp or conventional clamp from the same surgical incision. We fixed Chitwood clamp with a silk stay suture to obviate any hindrance by clamp in the surgical field.

7. We used antegrade del Nido cardioplegia for myocardial preservation. Cardioplegia cannula was the same as in conventional surgery (Edward life sciences/Medtronic).

8. RA was opened parallel to the atrioventricular groove, from RA appendage toward IVC.

9. For better VSD exposure, we used pledgeted sutures along the margins of VSD. Every pledgeted suture worked as a stay suture also that helped in exposing VSD margins gradually and sequentially. VSD was 
examined, and closure was performed in a routine fashion using horizontal mattress pledgeted polyester sutures, with double velour Dacron (DVD) patch (Fig. 1b).

10. De-airing was done through left atrium vent and aortic root venting prior to gradually weaning off bypass. Patients were electively ventilated for 3-4 h.

11. For subpulmonic VSD, we did left anterolateral thoracotomy and opened second intercostal space. From here first we put aortic cannula and a single venous cannula in right atrium to go on bypass to decompress the heart. After decompressing the heart, we could access the SVC/IVC easily. Closure of VSD was done through right ventriculotomy.

12. On intraoperative echocardiography, mild aortic cusp prolapse was noted in 2 patients (missed on preoperative echo). After repair of VSD, there was no significant aortic regurgitation on echo assessment, so we did not repair or replace the aortic valve.

13. We put single ventricular and single atrial pacing wire for atrioventricular pacing. Pericardium was closed above aorta and partially above RA; rest pericardium was left open to drain the pericardial cavity into the thoracic cavity. We put a single chest drain to drain the thoracic as well as pericardial cavity.

\section{Data analysis}

This is a retrospective, observational study of all cases undergoing VSD correction during November 2016 to November 2018. Data were entered in Microsoft Excel software. Continuous variables were expressed as mean $\pm \mathrm{SD}$, and categorical data were expressed as proportions. In our study, there was no control or comparison group. Demographics, intraoperative data, postoperative morbidity and mortality, and follow-up were analyzed.

Table 2 Intraoperative variables

\begin{tabular}{ll}
\hline Variable & Mean \pm SD (range) or no \\
\hline Incision length (cm) & $07.16 \pm 02.08(5-9)$ \\
Aortic clamp time (min) & $38.51 \pm 13.08(26-56)$ \\
Cardiopulmonary bypass time (min) & $61.72 \pm 14.20(48-78)$ \\
Additional procedures & \\
1. Atrial septal defect closure & 4 \\
2. Tricuspid anteroseptal commissural plasty & 2 \\
3. Aortic cusp repair & 2 \\
\hline
\end{tabular}

Continuous variables expressed as mean \pm standard deviation

\section{Results}

Cardiac defects were corrected in all patients through anterolateral thoracotomy (Table 2). The mean incision length was $7.16 \pm 2.08 \mathrm{~cm}$ (range, 5 to $9 \mathrm{~cm}$ ). Cardiopulmonary bypass time was $61.72 \pm 14.20 \mathrm{~min}$ (range, 48 to $78 \mathrm{~min}$ ), and aortic clamp time was $38.51 \pm 13.08 \mathrm{~min}$ (range, 26 to $56 \mathrm{~min}$ ). Patients were discharged on 4th to 7 th post-op day (mean $4.92 \pm 1.80$ day). Every patient was satisfied with the surgical scar and resumed routine work in 1-month follow-up (assessed by pre-formed subjective questionnaire). No residual shunt was detected on echocardiography before discharge, or in the follow-up.

\section{Early or primary results}

There was no early postoperative mortality. There were no atrio-ventricular (AV) blocks, residual shunt, pericardial tamponade, reoperation for bleeding, and no complications related to the incision (Table 3). The mean blood transfusion requirement was $0.40 \pm 1.32$ units. Mean ICU stay was $2.13 \pm 0.32$ day, and hospital stay was $4.92 \pm 1.80$ day (range 4 to 7 days).

\section{Late or secondary results}

There was no incidence of deep sternal wound infection; only one patient had minor serous discharge that recovered well on next follow-up. Postoperative scars were cosmetically excellent (assessed by pre-formed subjective questionnaire), and it was totally hidden under sub-mammary fold without a chest deformity (Fig. 2). No late mortality was recorded in first 30day follow-up.

Table 3 Postoperative data

\begin{tabular}{ll}
\hline Outcomes & Mean \pm SD (range) or no \\
\hline Hospital mortality & Nil \\
Low output syndrome & Nil \\
Re-exploration for bleeding & Nil \\
Blood transfusion (units) & $0.40 \pm 1.36$ \\
Fever & 2 \\
Mediastinal bleeding & Nil \\
Acute renal failure & Nil \\
Mediastinitis & Nil \\
ICU stay (days) & $1.83 \pm 1.32$ \\
Hospital stay (days) & $4.92 \pm 1.80$ \\
Wound infection & Nil \\
Dehiscence & Nil \\
30-day mortality & Nil \\
\hline
\end{tabular}




\section{Follow-up}

First and second follow-ups were fixed at 7th and 30th day after discharge. Follow-up was performed in outdoor by personal contact or by phone contact with patients and family members, with supplemental information supplied by family physicians and referring cardiologists.

On follow-up, 2-D echo and clinical assessment for cardiac functions were done as a routine protocol. Routine chest X-ray and blood investigations were done as and when required.

\section{Discussion}

Surgical correction for simple congenital heart disease has changed noticeably in last decade during which different surgical techniques have been developed. We analyzed the clinical data of 54 patients who underwent repair of VSDs via anterolateral thoracotomy with central cannulation. The benefits of anterolateral thoracotomy are good cosmetic appearance, decrease in the chance of keloid and hypertrophic scar, easier management of wound infection, and early mobilization $[6,7]$. Short hospital stay, early discharge, and early return to daily activity also save a lot of health expenditure of the patient, which matters a lot in our country. In addition, adverse psychological consequences of unaesthetic scarring on young boys and girls are more significant than in adult patients, making our approach popular. Cosmetic results of the surgical approach are important, especially in girls and women in India as surgical scar is a marriage taboo [8].

As a result, nonsurgical trans-catheter interventions with minimal invasiveness and favorable cosmetic results are more popular with patients, sometimes even at the cost of suboptimal results $[9,10]$. In addition, VSDs can also be repaired robotically via a minimally invasive access [11]. To increase success rates, improve cosmetic results, and reduce surgical trauma, minimally invasive cardiac surgery has been increasingly applied in the treatment of VSDs. However there is a great paucity of relevant literature.

Mishaly et al. [5] in 2008 published data of 75 patients in which patients underwent right anterior minithoracotomy approach for congenital cardiac defect repair. In his series, peripheral cannulation was done, while in our series, we adopted central cannulation via thoracotomy incision. A group from Beijing (Liu et al.) published their experience of 488 patients in repairing, through thoracotomies, more complex defects (e.g., tetralogy of Fallot) in children and adolescents [12]. Wang et al. reported 274 patients with ventricular septal defects, repaired via a minimal right vertical infra-axillary thoracotomy [4]. In their study, there were no deaths or complications from the infection of incisional wound, arrhythmias, and no significant differences in CPB time or postoperative ventilator time. The results were comparable to our study.
Yamada et al. [13] compared early postoperative quality of life in minithoracotomy and conventional sternotomy, with results same as our series. Most of these series adopted a policy of peripheral cannulation that was different from our series.

Technical challenges like central aortic cannulation, asymmetric breast development, defibrillation, and difficult deairing from the ventricle are well documented. In 2018 Hong et al. [14] published data of 182 patients, showing that the surgical success rate of VSD repair via right sub-mammary thoracotomy and right vertical infra-axillary thoracotomy was similar to median sternotomy, which means all the three methods can achieve satisfactory clinical results. Based on their findings, they recommended that right sub-mammary thoracotomy and right vertical infra-axillary thoracotomy be used as effective and safe alternatives in VSD surgical repair. In this series, they adopted same policy as our series, i.e., cannulation and cross clamp from same incision in group B of their study, and results are similar to our study.

Gaetano et al. [7] from Italy published a series of 132 patients with similar policy as in our series of anterolateral thoracotomy with central cannulation technique for radical correction of congenital heart disease and had similar results. For female patients, Jung et al. [15] advocated the anterolateral minithoracotomy as the incision access, quite akin to our protocols.

However, few reports suggested that this incision may dissect the breast tissue and result in asymmetrical development and a decrease in sensitivity of the nipple. We avoided these side effects by adopting a modified incision, whereby the incision was in the sub-mammary fold (Figs. 2 and 3). In addition, we also followed the rule that the incision should be at least 2 to $3 \mathrm{~cm}$ apart and below from the mammary areola in patients with undeveloped breasts. In the present anterolateral

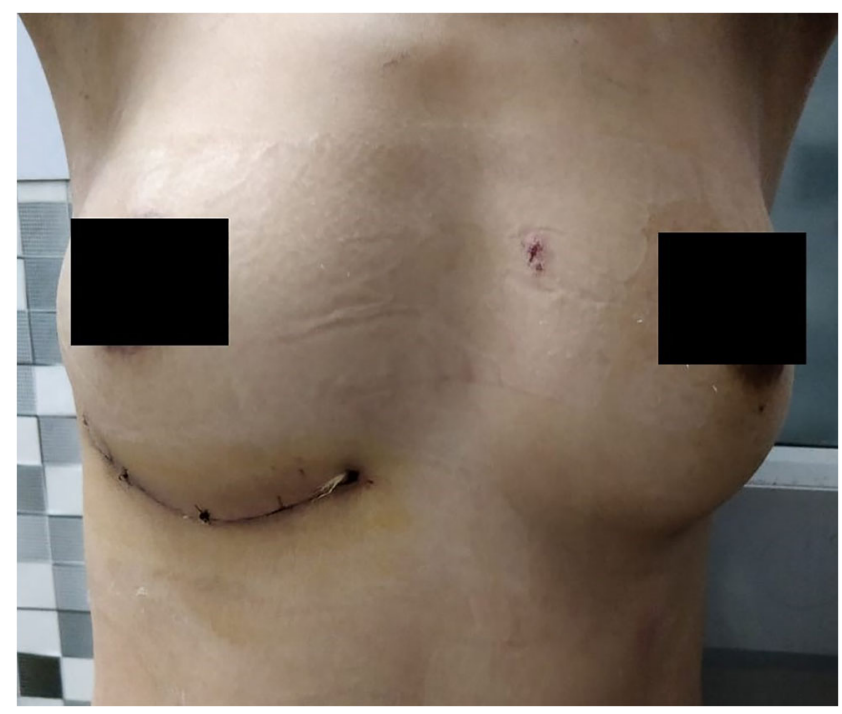

Fig. 3 Postoperative surgical scar 
thoracotomy series, the incision ranged from 5 to $9 \mathrm{~cm}$. To avoid distortion of the growing breast tissue, particularly in the prepubescent girls, we tried to make incision as small as possible and as low as the fifth intercostal space, below the future development of the breast tissue, which would not impede the growth of the breast tissue and the pectoralis muscle (Fig. 3).

A sternal wound, once infected, becomes a challenge for the cardiac surgeon to treat it. It usually leaves a big scar and an awkward appearance. The incidence of wound infections and septic complications is minimal with a thoracotomy than with a median sternotomy; this virtually eliminates mediastinitis [16-18]. We believe that an almost obscure, small, lower anterolateral thoracotomy scar in the skin fold of breast is more cosmetic and patient friendly as it provides early and fast recovery, less ICU stay, and decreased chances of mediastinitis, as compared with sternal incision. The other most important aspect of this approach is central cannulation which obviates the complications associated with peripheral cannulation. Our approach provides excellent exposure of the right atrium, both caval veins and the ascending aorta, almost in the same manner as via a median sternotomy. It exposes the defect just as the median sternotomy does, and the method of correction is the same. Our series allowed for a predictable exposure and improved cosmetics (Fig. 2). Moreover, it does not require special instruments. In India where surgical cost matters for poor patients, this approach is a good and safe alternative. According to analytic results of our series, all the patients obtained fast postoperative recovery and less ICU stay (Table 3). Very few series are available worldwide in which thoracotomy approach with central cannulation is adopted for VSD repair. Our series opens up path for new researchers, especially in India, where resources are limited and cost of surgery is always a big issue. As far as the learning curve of a cardiac surgeon is concerned, if this approach is adopted as a routine part of a surgical unit, then new budding surgeons will definitely learn a lot. Initially our unit adopted a policy that we would take cases with favorable anatomy showed by 2-D echo. VSD associated with complex congenital variants were difficult to approach; that is why we put that cases in exclusion criteria or contraindications or high-risk cases.

\section{Limitations of our study}

Our study was a retrospective observational study and was limited by the number of cases and the fact that it was done in a single center. As we analyzed only collected data of operated patients and we did not compare with any control group, absence of control group is limitation of our study. Prospective randomized controlled studies with a larger sample size, even multicenter cooperation, must be conducted to confirm the results. In addition, a longer follow-up is essential, especially for those patients with thoracic deformity.

\section{Conclusion}

Anterolateral thoracotomy with conventional central cannulation can be a safe and good alternative to the conventional median sternotomy with good cosmetic results for the repair of VSDs.

Funding Nil

\section{Compliance with ethical standards}

Conflict of interest The authors declare that they have no conflicts of interest.

Ethical approval Institutional ethical committee approval was obtained for the study.

Human and animal rights and informed consent Informed consent was taken from the patient or the parents, in the case of children. No animals involved. Human rights protected according to Helsinki Declaration.

\section{References}

1. Hopkins RA, Bert AA, Buchholz B, Guarino K, Meyers M. Surgical patch closure of atrial septal defects. Ann Thorac Surg. 2004;77:2144-9.

2. Nicholson IA, Bichell DP, Bacha EA, del Nido PJ. Minimal sternotomy approach for congenital heart operations. Ann Thorac Surg. 2001;71:469-72.

3. Komai H, Naito Y, Fujiwara K, Noguchi Y. Cosmetic benefits of lower midline skin incision for pediatric open heart operation. A review of 100 cases. Jpn J Thorac Cardiovasc Surg. 2002;50:55-8.

4. Wang Q, Li Q, Zhang J, Wu Z, Zhou Q, Wang DJ. Ventricular septal defects closure using a minimal right vertical infraaxillary thoracotomy: seven-year experience in 274 patients. Ann Thoracic Surg. 2010;89:552-5.

5. Mishaly D, Ghosh P, Preisman S. Minimally invasive congenital cardiac surgery through right anterior minithoracotomy approach. Ann Thorac Surg. 2008;85:831-5.

6. Yoshimura N, Yamaguchi M, Oshima Y, Oka S, Ootaki Y, Yoshida M. Repair of atrial septal defect through a right posterolateral thoracotomy: a cosmetic approach for female patients. Ann Thorac Surg. 2001;72:2103-5.

7. Gaetano P, Raffaele G, Veronica R, et al. Anterolateral minithoracotomies for the radical correction of congenital heart defects. Tex Heart Inst J. 2009;36:575-9.

8. Lukram S, Sharma M, Sharan A, Dixit S, Sharma A. Submammary incision as right mini thoracotomy approach with intercostal nerve exclusion and blockage technique : a comparative study in the young Indian female population undergoing mitral valve replacement. Heart India. 2016;4:61-6.

9. Du ZD, Hijazi ZM, Kleinman CS, Silverman NH, Larntz K. Comparison between transcatheter and surgical closure of secundum atrial septal defect in children and adults: results of a multicenter nonrandomized trial. J Am Coll Cardiol. 2002;39: $1836-44$. 
10. Butera G, Carminati M, Chessa M, et al. Percutaneous versus surgical closure of secundum atrial septal defect: comparison of early results and complications. Am Heart J. 2006;151:228-234.

11. Gao C, Yang M, Wang G, Xiao C, Wang J, Zhao Y. Totally endoscopic robotic ventricular septal defect repair in the adult. J Thorac Cardiovasc Surg. 2012;144:1404-7.

12. Liu YL, Zhang HJ, Sun HS, et al. Repair of cardiac defects through a shorter right lateral thoracotomy in children. Ann Thorac Surg. 2000;70:738-41.

13. Yamada T, Ochiai R, Takeda J, Shin H, Yozu R. Comparison of early postoperative quality of life in minimally invasive versus conventional valve surgery. J Anesth. 2003;17:171-6.

14. Hong ZN, Chen Q, Lin ZW, et al. Surgical repair via submammary thoracotomy, right axillary thoracotomy and median sternotomy for ventricular septal defects. J Cardiothorac Surg. 2018;13:47.

15. Jung SH, Gon Je H, Choo SJ, Yun TJ, Chung CH, Lee JW. Right or left anterolateral minithoracotomy for repair of congenital ventricular septal defects in adult patients. Interact CardiovascThorac Surg. 2010;10:22-6.

16. Bauer M, Alexi-Meskishvilli V, Nakic Z, et al. The correction of congenital heart defects with less invasive approaches. Thorac Cardiovasc Surg. 2000;48:67-71.

17. Bigdelian H, Sedighi M, Movahedi F. Repair of atrial septal defect via right submammary minithoracotomy in children. Int Cardiovasc Res J. 2015, 2015;9:52-4.

18. Yaliniz H, Topcuoglu MS, Gocen U, et al. Comparison between minimal right vertical infra-axillary thoracotomy and standard median sternotomy for repair of atrial septal defects. Asian J Surg. 2015;38:199-204.

Publisher's note Springer Nature remains neutral with regard to jurisdictional claims in published maps and institutional affiliations. 